\title{
Does an agent's touch always matter? Study on virtual Midas touch, masculinity, social status, and compliance in Polish men
}

\author{
Justyna Świdrak ${ }^{1,2}$ (i) $\cdot$ Grzegorz Pochwatko $^{1}$ (D) $\cdot$ Andrea Insabato $^{3}$ (i)
}

Received: 31 October 2019 / Accepted: 29 October 2020 / Published online: 3 January 2021

(c) Springer Nature Switzerland AG 2021

\begin{abstract}
Traditional gender roles that define what is feminine and masculine also imply that men have higher social status than women. These stereotypes still influence how people interact with each other and with computers. Touch behaviour, essential in social interactions, is an interesting example of such social behaviours. The Midas touch effect describes a situation when a brief touch is used to influence one's behaviour. Our study aimed to analyse the influence of virtual touch on compliance in men in a decision-making game called Ultimatum. In a series of three studies, we investigated whether social cues such as gender, stereotypical masculine/feminine appearance, and high/low social status modify compliance to offers from embodied agents. We built an immersive version of a repeated Ultimatum game in which a proposer offers how to split ten coins, and a responder accepts or rejects the offer. In study 1, men and women played with a female and a male agent. In study 2 and 3 , men played with four agents each, differing in gender and levels of stereotypically seen masculinity and social status. There was no significant touch effect. Compliance was secured mostly by the value of the offer: the more generous the offer, the higher the compliance rate. We also found evidence for the perceived masculinity and social status influence. We also describe relationships between agents' characteristics and the perception of their touch. The results are discussed in the context of social characteristics that are important in agent design and the effectiveness of social influence techniques in virtual reality.
\end{abstract}

Keywords Virtual touch $\cdot$ Embodied agents $\cdot$ Social influence $\cdot$ Masculinity $\cdot$ Social status

\section{Virtual Midas touch}

Gender roles visibly influence how people interact with each other, both verbally and nonverbally [1]. Our study aimed to

Electronic supplementary material The online version of this article (https://doi.org/10.1007/s12193-020-00351-x) contains

supplementary material, which is available to authorized users.

Justyna Świdrak

jswidrak@psych.pan.pl

Grzegorz Pochwatko

grzegorz.pochwatko@psych.pan.pl

Andrea Insabato

insabato@clinic.cat

1 Institute of Psychology, Polish Academy of Sciences, Warsaw, Poland

2 Experimental Virtual Environments for Neuroscience and Technology, Faculty of Psychology, University of Barcelona, Barcelona, Spain

3 August Pi and Sunyer Biomedical Research Institute (IDIBAPS), Barcelona, Spain understand how they modify the influence of touch (called the Midas touch effect) of embodied agents. Traditional masculinity is associated with toughness, physical strength, dominance, and financial success. It also implies rejection or even hostility towards anything associated with femininity, which may sometimes lead to violence; for example, towards non-heteronormative men or men with more effeminate characteristics [2]. On the other hand, femininity is traditionally related to warmness and submissiveness [3]. A woman who does not follow these roles may face criticism for being selfish, overly masculine, or aggressive [4]. These stereotypes have shaped societies for centuries and still penetrate invisibly interpersonal communication, including mediated human-human and human-computer communication. For example, we know that "the gender stereotypes people have for individual tasks and social roles influence their preference for agent form" [5, 14], and in a Japanese study on perceived femininity and masculinity in agents, men and women preferred respectively feminine female and masculine male agents [6]. 
Touch behaviour, essential in social interactions, is also subject to gender norms. Henley [7] observed that men initiate one-way touch more frequently than women, which reflects masculine control and dominance (e.g. the supervisor touching the shoulder of his secretary). Moreover, men feel uncomfortable being touched by another man and avoid same-gender touch, which is associated with male homophobia $[8,9]$.

Differences in touching behaviours are also reflected in the efficiency of the Midas touch effect, a social influence technique which describes a situation when a light touch of a shoulder or arm increases chances for obtaining spontaneous help or complying with a request [10]. It is most efficient when the request is relatively straightforward [11] — for example, customers who were touched on their shoulder tend to tip more [12]. The efficiency of this technique may be modified by the characteristics of the touching and the touched. Women's touch seems to be more influential than men's [13]. Sometimes, the strongest effect was obtained when the interaction occurred between people of opposite genders [14]. There is also some evidence that high-social-status cues (such as clothing, profession) influence the Midas touch effect [15]; nevertheless, it is important to take Guéguen's work (as the only author) with caution in the light of recent criticism [16]. In Poland, a highly homophobic country [17], women and men complied to a request of a female confederate more frequently when she added a touch than when she did not. Interestingly, this effect was reversed when both the confederate and the participant were men - in such a situation, a touch accompanying the request significantly reduced compliance compared to a no-touch request. This effect was linked to strong male homophobia $[18,19]$.

Another factor influencing the efficiency of the Midas touch effect is the social context of the interaction. For example, compared with a supportive environment, touch in a competitive environment may reduce helping behaviour [20]. The influence of cooperative $v s$ collaborative situations on touch was also investigated in the mediated touch. This type of touch has recently drawn particular attention of researchers due to the COVID-19 pandemic, which has restricted drastically human physical contact [21]. Some early studies showed, for example, that embodied agents that used touch were seen as warmer than non-touching agents, while no effect of the situation was found [22]. Touch also increases human likeness of embodied agents [23]. One of the wellknown paradigms to study collaboration and competition is the Ultimatum game [24]. In this game, two players need to divide between each other a given pie (for example, some amount of money). One of them, the proposer, offers the split and the other one, a responder, can either accept it or reject it. When the offer is accepted, both players gain the agreed amount. Rejection means none of them gets anything. This seemingly straightforward paradigm has been used in multi- ple studies, including human-computer interaction [25, 26]. A repeated Ultimatum game was also used as a measurement of compliance in the mediated Midas touch effect [27]. The authors found a significant effect of touch on compliance compared with no stimulus, but no significant effect compared with a sound. A similar effect was demonstrated using immersive virtual reality, where a touch initiated by an embodied agent increased compliance in men [28].

Influential embodied agents play a crucial role in gaming and in any situation where the presence of a human is not safe or feasible, for instance in military or medical training. In these situations, the level of social influence elicited by an agent directly impacts the performance level of the user. Touch may serve as a useful way to influence user behaviour or draw their attention.

Nevertheless, human-agent touch remains understudied and underdeveloped in comparison to the visual and auditory elements of human-agent communication [29] which creates an urgent need to investigate the dynamics and factors influencing touch between humans and agents. Here, we present a series of three studies which aim to understand better the influence of embodied agents' touch and traits in a virtual Ultimatum game. The research questions and hypotheses are the following.

Q1. Do people perceive a touch of various agents differently?

H1. Perception of an agent's masculinity and social status will influence the evaluation of the agent's touch:

H1a. A female agent's touch will be perceived more positively than a male agent's touch. (study 1).

H1b. A lower masculinity of the male agent will result in a more negative perception of touch. (study 2).

H1c. The higher the male agent's social status, the more positive the agent's touch will be perceived. (study 3 ).

\section{Q2. Does gender influence the efficiency of the Midas touch? (study 1)}

H2.1 Touch will evoke higher compliance than no stimulus or a buzz.

H2.2 Compared to trials with no stimulus or a buzz, the efficiency of touch will be moderated by the participant's and the agent's gender:

H2.2a. Women will be more prone to accept offers when touched by both agents.

H2.2b. Men will be less prone to accept offers when touched by the male agent and more prone to accept it when touched by a female agent. 
Q3. Does stereotypical masculinity/femininity of the agent influence the efficiency of the Midas touch effect? (study 2)

H3 Compared to trials with no stimulus or a buzz, the efficiency of touch will depend on the masculinity/femininity of the agents:

H3a. A feminine female agent's touch will evoke the highest compliance.

H3b. The effeminate male agent's touch will evoke the lowest compliance.

Q4. Does the social status of the agent influence the efficiency of the Midas touch? (study 3)

H4 Compared to trials with no stimulus or a buzz, the highstatus agent's touch will evoke higher compliance to touch than low-status agents.

The method section contains a joint description of the procedure in all studies, while the results section reports data from each study separately. In the last part of the manuscript, we summarise and discuss the results. We also mention several shortcomings and propose future directions for studies.

\section{Method}

\subsection{Study design}

Study 1 had a mixed design: 2 PARTICIPANT $($ female $/$ male $) \times 2 \quad$ AGENT $($ female $/$ male $) \times 3 \quad$ ACTION (none/buzz/touch). In study 2, we applied a 2 AGENT $($ female/male) $\times 2$ STEREOTYPE (masculine/feminine) $\times$ 3 ACTION (none/buzz/touch) within-subject design with repeated measures. In study 3 , we used the same design, replacing STEREOTYPE with STATUS (high/low). In study 1 , women and men played with two agents each, one female and one male; the game consisted of 40 trials, out of which the participant played 20 trials as a proposer. In studies 2 and 3 only men participated; they played with four agents, two females and two males; each game consisted of 88 trials, out of which the participant played 48 as the responder, equally distributed between the agents. In study 1 , the order of trials was fully random, in study 2 and 3 the participant always played the first round as the proposer, so that they could familiarise themselves with the interface of the game.

\subsection{Sample}

Data collection took place in Warsaw (Poland), from May 2016 to March 2017. In study 1, we recruited women and men, while in study 2 and 3, we recruited only men between
18 and 35 years old with no physical nor psychological disorders. It was possible to participate only in one study. After we informed participants about the possible negative consequences of using a virtual reality (VR) headset (e.g. dizziness, headache, nausea), they signed an informed consent form. None of the participants had previous experience with VR. Every participant was rewarded at the end of the study with one (study 1) or two (study 2 and 3 ) cinema vouchers worth $\sim 5 €$ each. In the first study, 19 women aged 19-33 $(M=23, \mathrm{SD}=3)$ and 15 men aged $21-33(M=27, S D$ $=3$ ) participated. In the second study 40 men aged 18-33 $(M=23, S D=4)$ participated, and in the third one, 39 men aged 19-34 $(M=24, S D=4)$ participated. In study 2 and 3, we decided to recruit only men due to gender differences in touch behaviours and homophobia in Poland [19]. Because of these differences, our hypotheses would not be relevant for female participants. Most of the participants had graduated high school (study 1: $54.5 \%$, study $2: 77.5 \%$, study 3 : $51.3 \%)$ or university $(36 \%, 22.5 \%$, and $38.5 \%$ respectively). We obtained ethical approval from the Ethics Committee of the Institute of Psychology, Polish Academy of Sciences.

\subsection{Measures}

\subsubsection{Behaviour}

Compliance was measured by counting accepted by the participant offers $(0$-rejected, 1 -accepted $)$.

\subsubsection{Self-report measures}

In the post-experimental questionnaire, we tested the perception of touch and the agents. First, participants saw a picture of the agent in randomised order, which was followed by two questions: 1. In your opinion, how feminine/masculine is this person? (perceived masculinity) (0-very feminine to 100-very masculine); 2 . In your opinion, how low/high on a social ladder is this person? (perceived social status) (0-very low to 100—very high). Participants responded using a visual analogue scale.

Touch perception of each agent was measured by asking, Please indicate, how much the touch of (name) was for you. There were nine adjectives (natural, surprising, pleasant, weak, adequate, well synchronised with the animation, strange, frightening, nice) which participants assessed on a 5-point Likert scale from 1-totally disagree to 5-totally agree. All questionnaires were in Polish. 


\subsection{Procedure}

\subsubsection{Overall procedure}

The general procedure was identical in all experiments. The participant came to the laboratory at the agreed time where the experimenter welcomed them, informed about the procedure and potential health risks, and asked them to sign the informed consent form. Next, they entered the laboratory where the experimenter helped them with the placement of the bioelectrodes ${ }^{1}$ and donning the head-mounted display (HDM), headphones, and vibrating band on the left arm (in the middle between the elbow and shoulder, on a t-shirt's sleeve if possible), which was fixed with a Velcro strap. After testing the signal and recording the baseline for $5 \mathrm{~min}$, participants entered the virtual environment. At the beginning of the game, the experimenter explained how to use the game interface and then left the laboratory. When the game was over, she returned and helped the participant to remove the bioelectrodes, HMD, and vibrating band, and asked the participant to fill in the questionnaires displayed on a computer screen. The experimenter monitored the participant by streaming the virtual environment and psychophysiological signals to the second room.

For safety reasons, the participant was advised that the door will remain open and, in case of feeling unwell/experiencing any inconvenience/having doubts about the procedure, that they should immediately call the experimenter, who was in a room in the immediate vicinity of the laboratory. Every 10-15 min or in case of inactivity/strange behaviour, the experimenter checked on the participant. The whole procedure lasted around $90 \mathrm{~min}$, including $20 \mathrm{~min}$ in study 1 and $\sim 40$ min in study 2 and 3 inside the virtual Ultimatum game. None of the participants complained about feeling unwell, the length of the procedure in VR or any other problems. This effect was obtained through the VR design, mainly reduced light stimulation and a relatively simple, static environment. Moreover, no evidence was found that the VR optical arrangement affected the binocular status of the eyes in a 40-min-long exposure [30].

In the end, the experimenter debriefed the participant and rewarded them. Due to a more prolonged procedure in study 2 and 3, whether the participant received one or two cinema vouchers depended on the number of coins collected during the game. Participants who gained more than 200 coins received two tickets (more than $50 \%$ of participants). Partic-

\footnotetext{
${ }^{1}$ In all studies, the skin conductance, heart rate, and electromyographical data were recorded. We decided to exclude these results from the manuscript due to problems with the signal quality, lack of important effects, and space limitations of the manuscript. Data can be obtained from the corresponding author. HR analysis can be found in X, Y. (2019).
}

ipants did not know the threshold for receiving two tickets before the game.

\subsubsection{Ultimatum game}

The Ultimatum game was built by Blue Brick ${ }^{2}$ in Unity 2016, while the 3D models of agents were designed in MakeHuman. Participants used a keyboard in study 1 and Xbox controllers in study 2 and 3 to navigate through the environment. They could feel touch while seeing the agent leaning towards them and reaching with their hand to the place where participant's real left arm was. Agents were moving delicately, but they did not interact with participants otherwise - they had no facial expression and did not talk, but they did maintain eye contact randomly in 70/30 ratio (see video).

Due to technical limitations, participants did not have a virtual body, which is why they could "touch" the agent by pressing a button in the menu before making an offer. In study 1 , we were interested in gender differences between women and men and their compliance to touch of a female and male agent. We designed two stereotypical, averagely attractive female and male Caucasian agents (Table 1).

Having observed several procedural problems in study 1 , we applied some changes in the game in study 2 and $3 .^{3}$ First, we changed the sound to a more delicate one, because several participants complained about it. Second, we changed the keyboard to an Xbox controller to make the interface more user-friendly and intuitive. We limited the full trials randomness by adding a rule that in the first trial, the participant always plays as a proposer to make sure he has time to learn the interface. In study 2 , we manipulated the stereotypical femininity/masculinity traits, such as haircut, height, size of the jaw, waist, and shoulders. In study 3, we manipulated the characteristics stereotypically associated with high and low social status, the outfit and haircut (Table 1).

Agents characteristics selection 3D models, names, and professions for all agents were selected in an online survey to make sure they match the stereotype. We presented in a randomised order 28 various models of both genders, which online volunteers rated on their attractiveness, masculinity, and social status levels on a $0-100$ scale. Then they rated, in the same way, a list of 57 male and 36 female names and 47 professions. In the survey participated 38 Polish people (including 18 women). Based on these results, we selected the following models (Table 1).

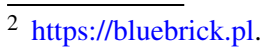

${ }^{3}$ Due to the budget and contract limitations, we were not able to remove some of the important limitations of the virtual environment design from study 1 .
} 
Table 1 Selected agents

\begin{tabular}{l}
\hline Experimental manipulation \\
\hline Study \\
Appearance \\
Feminine/effeminate \\
Masculine \\
High status
\end{tabular}

\subsubsection{Proposer and responder}

In the first step, the participant could see the agent, with which he was playing, sitting in front of him at a small table (Video). He could also read the name, the age, and the profession of the agents. As a proposer, after a few seconds, he could see the menu screen where he could choose an action: "none", "play a sound", or "touch". Below, he could also select the offer he wanted to make. The role of proposer served in our study to (1) hide the real aim of the study, and (2) make the game more interesting for the participant. Players had to split ten coins in any configuration from 1:9 (extremely generous) to 9:1 (extremely selfish). Several seconds after the decision (randomised value between 4 and $7 \mathrm{~s}$ ), the agent accepted or rejected the offer. In the role of a responder, the participant saw the agent, then was touched, or heard a buzz or just waited for a few seconds (also randomised) and at the end, saw the proposed offer which they could accept or reject. The number of trails with no stimulus, buzz, and touch was balanced in all studies.

After the acceptance, players gained the agreed amount of coins; in case of rejection, neither of them won anything. In the background of the environment, participants could see how many coins they gained so far with a given agent. In the final screen, they saw a summary with the total amount of gained coins.

\subsection{Apparatus and data analysis}

We carried out the studies on a computer with operational system Windows 10, the Intel Core i5-6500 Skylake processor and the MSI GeForce GTX 960 graphic card. The participant used Oculus Rift; the vibrating band consisted of a $3 \mathrm{~V}$ vibration motor placed using a Velcro band. It was connected to the Arduino Leonardo, which received the signal from the PC through a USB cable.

The manipulation check was tested using ANOVA (study 1) and Tukey's multiple comparisons test (study 2 and 3). In study 1 and 2, the dependent variable (DV) was the perceived masculinity/femininity, in study 3 , the perceived social status. In all studies, the independent variable (IV) was the agent.

We analysed touch perception in three steps: (1) We carried out a principal component analysis (PCA); (2) We tested differences for each study with ANOVA (study 1, IV: participant's gender, agent) and Tukey multiple comparisons test (study 2 and 3, IV: agent) using PCA factors as DV; 3. We calculated Pearson's $r$ correlations between obtained factors and masculinity (study 1 and 2) and status (study 3 ). 


\subsubsection{Compliance}

Analysis of compliance from all three experiments was built using logistic regression models in which the dependent variable was the acceptance of the offer $(1$-accepted, 0 -rejected). In study 1 , the predictors were the offer (standardised), the perceived masculinity (standardised), agent's gender (AG), participant's gender (PG), touch, sound and two interactions: $\mathrm{PG} \times$ masculinity and $\mathrm{PG} \times$ touch. The model in study 2 included the offer (standardised), the perceived masculinity (standardised), AG, touch, sound, and the AG $\times$ offer interaction. In the last study, the predictors were the offer (standardised), the perceived masculinity (standardised), the perceived social status (standardised), AG, touch, sound, and the status $\times$ offer interaction. Other interactions were also considered but discarded from the final model for being not statistically significant (widely variable and small average effect), not increasing the model's likelihood function or not being of interest. However, we kept all strong (statistically significant) interactions as well as interactions that are of interest, whether or not statistically significant [31]. The accuracy of each model was compared to that of a null model; that is, the proportion of accepted or rejected offers in the dataset, whichever was higher. This corresponds to a model with only the intercept term, thus a model that does not use any information from independent variables.

We estimated parameters from all models using data pooled from all subjects together. We then used crossvalidation to assess the robustness of all models to variations in the data. The data from these experiments require crossvalidation for grouped data since there is natural grouping by participant imposed by the repeated-measures design. In this case, random permutation cross-validation could produce training and test set with a correlation higher than would be expected by chance. Here we used a leave-one-participantout cross-validation, thus for data from each experiment we had $k$ folds where $k$ is the number of participants in that experiment. In each fold, we estimated parameters of the model using the training set composed of all but one participant and assessed the accuracy (proportion of correct predictions) on the test set composed of the trials from the held-out participant. In every fold, a different participant was used as a test set. To ensure that no information from the test set could influence the estimation of parameters, we considered the model as the full set of parameters, including both the logistic regression and variables transformations. Thus, the mean and standard deviation used for standardisation were estimated only using the training set.

\section{Results}

\subsection{Study 1}

\subsubsection{Agents' perception}

In study 1, we aimed to use a female and a male agent representing an average woman and man of equal social status (Table 1). ANOVA revealed significant differences in perceived masculinity $(F(1,70)=39.11, p<.001)$, with the male agent scoring on average $63.6(S D=16)$ and the female agent scoring $33.5(S D=23.8)$. The variances did not overlap; therefore, our manipulation was successful.

\subsubsection{Touch perception}

The PCA for the touch perception scale was used to reduce the number of factors. Bartlett's test of sphericity was significant $\left(\chi^{2}=736, p<.001\right)$ and the Kaiser-Meyer-Olkin test score was .72. We built the model with the "varimax" rotation. The PCA revealed three factors with eigenvalues $>1$, which we called pleasantness, awkwardness, and adequacy based on the item loadings (Appendix Table A1 and Figure A1). The three-factorial model explained $45 \%$ of the variance. H1a was verified by analysing the perception of touch in study 1 . Touch of a female agent was significantly more pleasant than that of a male agent $(F(1,66)=38.31, p<.001$, $\left.\eta^{2}=.36\right)$ equally for men and women $(p=.082)$. There was also a small effect of participant's gender in awkwardness, with women judging the touch of both agents as slightly more awkward than men $\left(F(1,66)=4.07, p=.047, \eta^{2}=.05\right)$. We found differences in the perception of the adequacy of touch. Additionally, for the female agent, the more masculine the agent was seen to be, the more adequate her touch felt (Pearson's $r=.50, p=.037$ ), while for the male agent no correlations between masculinity and the pleasantness, awkwardness, nor adequacy of touch were found. These results may partially confirm H1a: female agent's touch was seen as much more pleasant; how adequate her touch felt was also related to her perceived masculinity. We acknowledge though that the H1a was only partially confirmed because no relationships were found for the male agent.

\subsubsection{Compliance}

The pooled logistic regression model was built with 660 observations and eight degrees of freedom; the pseudo $R^{2}$ was .16. Model's prediction accuracy was high (.81, the accuracy of the null model was .76) when calculated using the full dataset. Across cross-validation folds, the accuracy was also higher than the null-model in the majority of folds (Table 2).

The most important predictor for accepting the offer was the offer's value, where an increase of one coin corresponded 
Table 2 Pooled logistic regression models of compliance

\begin{tabular}{|c|c|c|c|c|c|c|c|c|c|c|c|c|}
\hline \multirow{3}{*}{$\begin{array}{l}\text { Accepted } \\
\text { offers }\end{array}$} & \multicolumn{4}{|c|}{ Study 1 gender } & \multirow{2}{*}{\multicolumn{4}{|c|}{$\begin{array}{l}\text { Study } 2 \text { masculinity } \\
N=1914 M=78 \%\end{array}$}} & \multirow{2}{*}{\multicolumn{4}{|c|}{$\begin{array}{l}\text { Study } 3 \text { social status } \\
N=1965 M=67 \%\end{array}$}} \\
\hline & \multicolumn{4}{|c|}{$N=680 M=77 \%$} & & & & & & & & \\
\hline & Coef & $S E$ & $z$ & $p$ & Coef. & $S E$ & $z$ & $p$ & Coef. & $S E$ & $z$ & $p$ \\
\hline Intercept & 1.68 & 25 & 678 & $<.001$ & 2.00 & .17 & 11.73 & $<.001$ & .95 & .13 & 7.38 & $<.001$ \\
\hline Offer & .79 & .11 & 7.46 & $<.001$ & .99 & .14 & 13.84 & $<.001$ & 1.60 & .08 & 20.41 & $<.001$ \\
\hline Masculinity & y.12 & .15 & .81 & .420 & .13 & .08 & 1.74 & .083 & .14 & .08 & 1.69 & .091 \\
\hline Status & - & - & - & - & - & - & - & - & .17 & .07 & 2.55 & .011 \\
\hline AG & .16 & .20 & .76 & .445 & -.08 & .19 & -.42 & .676 & .32 & .18 & 1.91 & .056 \\
\hline PG & -.90 & .25 & -3.57 & $<.001$ & - & - & - & - & - & - & - & - \\
\hline Touch & -.38 & .32 & -1.18 & .237 & -.07 & .16 & -.47 & .670 & -.17 & .14 & -1.20 & .230 \\
\hline Sound & -.18 & .25 & -.74 & .462 & -.04 & .17 & -.25 & .805 & -.12 & .14 & -.82 & .410 \\
\hline $\begin{array}{l}\mathrm{PG} \times \\
\text { masculini }\end{array}$ & $\begin{array}{l}.89 \\
\text { ity }\end{array}$ & .25 & 3.61 & .000 & - & - & - & - & - & - & - & - \\
\hline $\begin{array}{l}\mathrm{PG} \times \\
\text { touch }\end{array}$ & .71 & .44 & 1.63 & .102 & - & - & - & - & - & - & - & - \\
\hline $\begin{array}{c}\mathrm{AG} \times \\
\text { offer }\end{array}$ & - & - & - & - & -.42 & .19 & -2.24 & .025 & - & - & - & - \\
\hline $\begin{array}{c}\text { Status } \times \\
\text { offer }\end{array}$ & - & - & - & - & - & - & - & - & -.15 & .08 & -1.95 & .051 \\
\hline
\end{tabular}

to a maximal difference in acceptance of almost $20 \%$ (coef. $=.79, S E=.10, p<.001)$. The touch effect was not significant, although interestingly, it had a different direction for men (negative) than for women (positive) (Fig. 1); nonetheless, we cannot confirm $\mathrm{H} 2.1$. As predicted in $\mathrm{H} 2.2 \mathrm{a}$, women accepted statistically more offers than men (coef. $=-.90$, $\mathrm{SE}=.25, p<.001)$. There was also a large effect of the interaction between the PG and the perceived masculinity of the agent. While the perceived masculinity did not affect women's choices, it strongly influenced acceptance in men (coef. $=.89, \mathrm{SE}=.25, p<.001$ ).

Therefore, even though the hypothesis $\mathrm{H} 2.2 \mathrm{~b}$ was not directly confirmed, we observed some evidence for the role of masculinity in male compliance. Leave-one-participant-out cross-validation shows that coefficients were stable to variations in the dataset (removal of all trials from one subject) (Fig. 2, for coefficient values across CV folds, see Appendix, Figure A2).

\subsection{Study 2}

\subsubsection{Agents' perception}

In study 2, participants played with agents that differed in stereotypical masculinity/femininity traits. Tukey test revealed significant $(p<.01)$ differences between all agents in both studies (Fig. 3). The largest difference was, as expected,

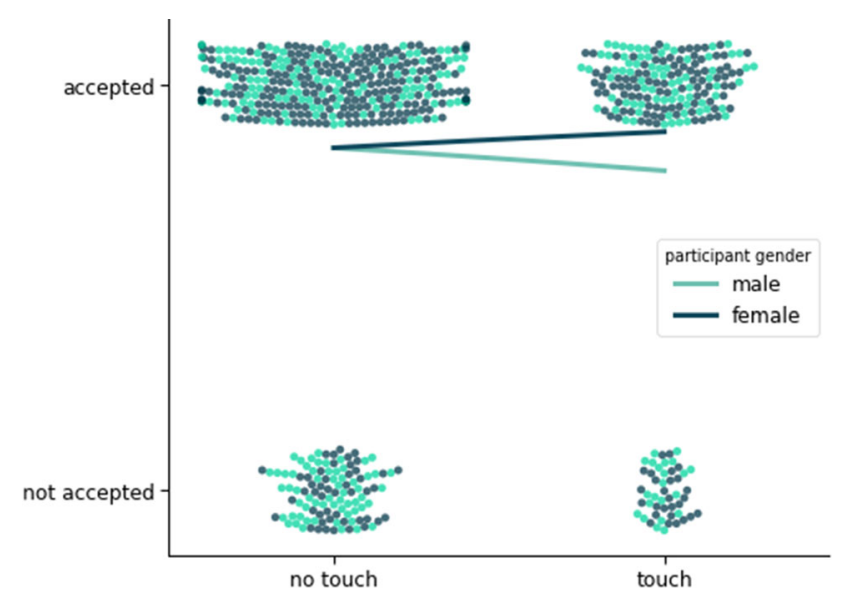

Fig. 1 Study 1. Offer acceptance in trials with touch compared to trials with no stimulus or sound

between the feminine female and masculine male agents, which refer to the stereotypical image of Caucasian man and woman (Mean difference $=69.70, S D=4.29$ ). On the other hand, the masculine female agent was rated as more masculine than the effeminate male agent (Mean difference $=7.60$, $\mathrm{SD}=4.32$ ).

Although the differences are in expected directions, there is considerable overlap among distributions. For this reason, we chose to use in the compliance model the perceived mas- 


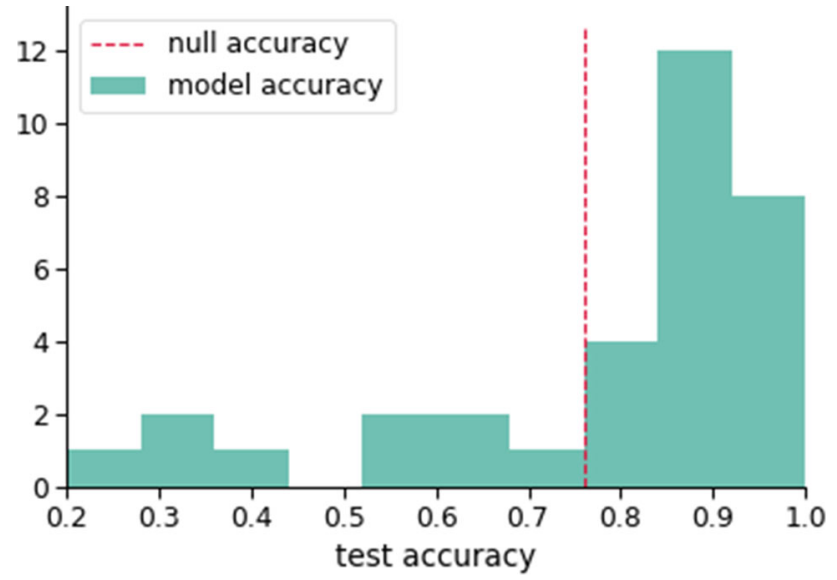

Fig. 2 Model accuracy in study 1

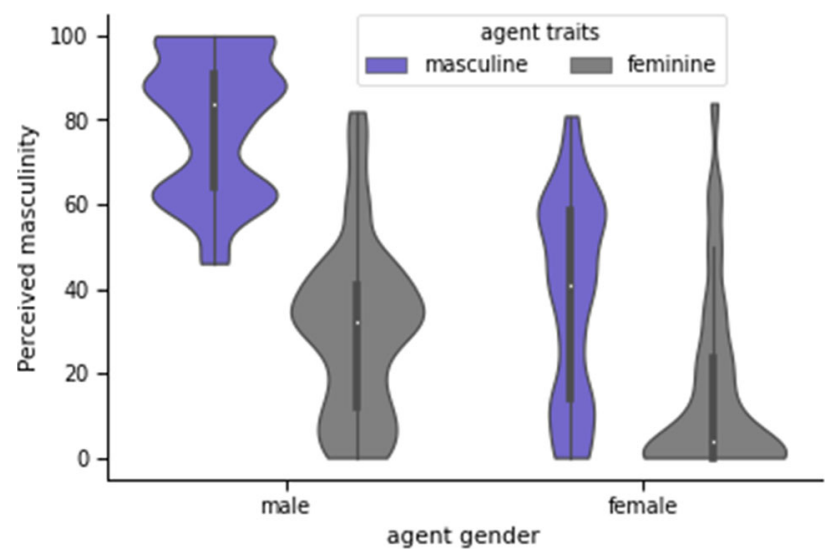

Fig. 3 Distribution of perceived masculinity for male and female agents in study 2

culinity instead of the dichotomous variable based on the study design.

\subsubsection{Touch perception}

We investigated further the relationship between masculinity and the perception of touch. In $\mathrm{H} 1 \mathrm{~b}$, we predicted that the masculinity of the male agents would be related to their touch perception. Tukey's test yielded the effeminate male agent' touch to be significantly less pleasant than the feminine female agent's (Mean diff. $=.85, \mathrm{SD}=.64, p=.001$ ) and from the masculine female agent's (Mean diff. $=.55, \mathrm{SD}=$ $.049, p=.02)$. Feminine female agent's touch was also significantly more pleasant than the masculine male agent's (Mean diff. $=.70, \mathrm{SD}=.49, p=.001)$. Moreover, the effeminate agent's touch scored on average lower on the adequacy and higher on the awkwardness than all the other agents; nevertheless, none of these differences was statistically significant, nor any correlations for the agents' masculinity level and the perception of their touch; therefore, H1b can be confirmed only partially.

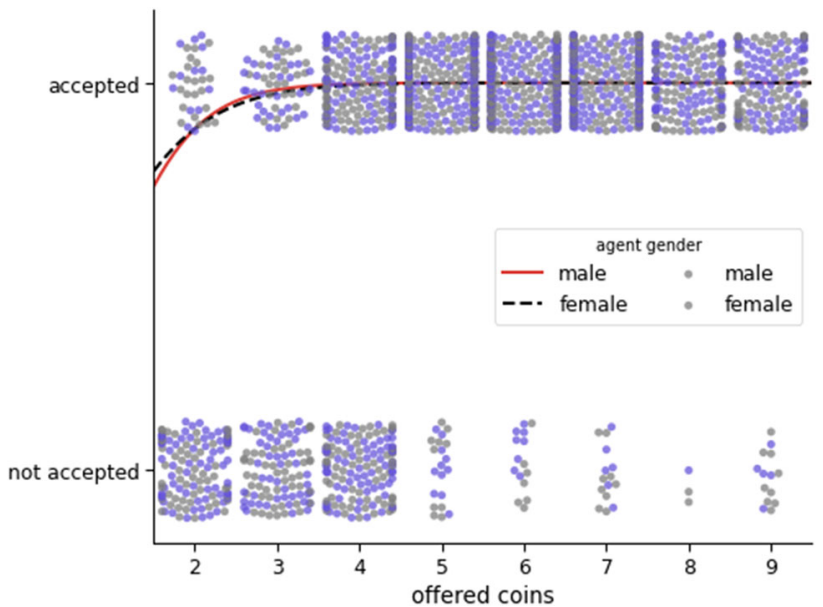

Fig. 4 Effect of the offer and the interaction with agent gender on compliance in study 2

\subsubsection{Compliance}

The pooled logistic regression model contained 1914 observations, six degrees of freedom, and the pseudo $R^{2}$ equalled .30 . The prediction accuracy of the model was .84 , while the accuracy of a null model was .77 (Table 2).

As in study 1, the offer's value influenced the acceptance strongly, with a maximal increase of chances of $50 \%$ (coef $=1.99, \mathrm{SE}=.14, p<.001$ ), although a large interactional effect of the offer and AG demonstrates that when playing with a female agent, the offer's value was less important than when playing with a male agent (coef. $=-.42, \mathrm{SE}=.19, p$ $=.025)$ (Fig. 4). Nevertheless, the main effect of gender was not significant.

There also was a trend suggesting that the perceived masculinity slightly increased the probability of accepting the offer (coef. $=.13, \mathrm{SE}=.08, p=.08$ ). We did not find any

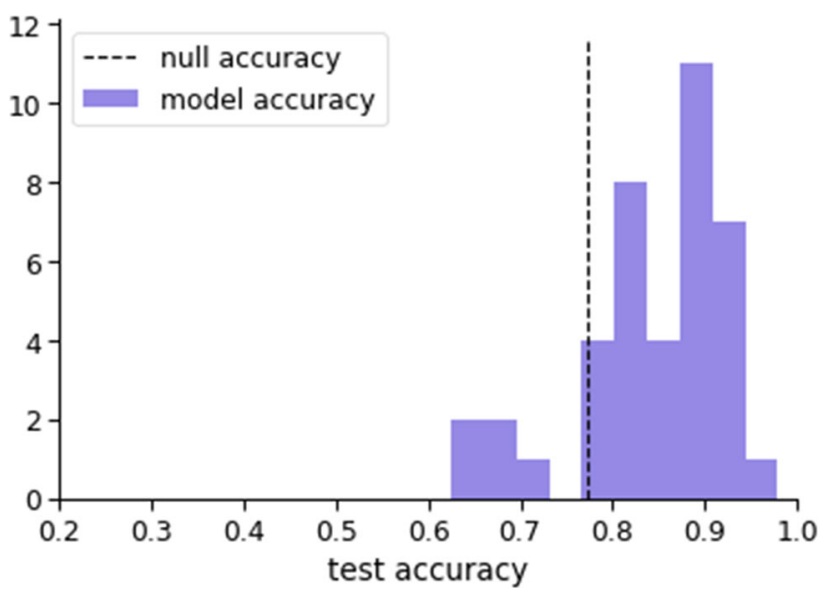

Fig. 5 Model accuracy in study 2 


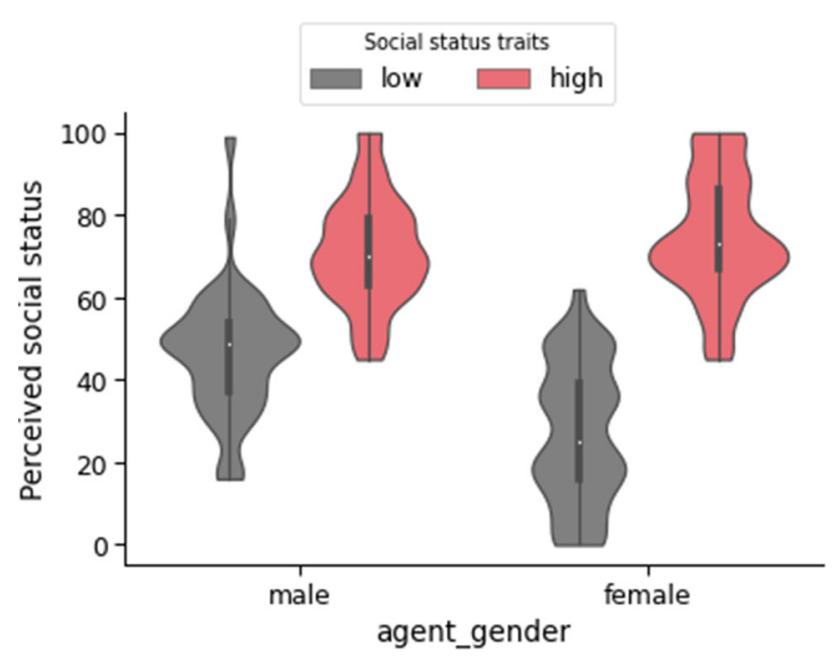

Fig. 6 Distribution of perceived social status for male and female agents in study 3

main nor interactional effect, neither of the touch nor the sound. Therefore, $\mathrm{H} 3 \mathrm{a}$ and $\mathrm{H} 3 \mathrm{~b}$ are not supported by the obtained results. Cross-validation showed that the patterns found on the whole dataset are stable across folds (Fig. 5, for coefficient values across $\mathrm{CV}$ folds, see Appendix Figure A3).

\subsection{Study 3}

\subsubsection{Agents' perception}

In study 3, all agents differed from each other $(p<.005)$, the largest mean difference was found between the female agents (Mean difference $=47.27, \mathrm{SD}=2.98$ ) in the expected direction (Fig. 6). The smallest difference was found between the low-status male and high-status female agent, with a mean difference equal to 3.19 ( $\mathrm{SD}=2.46$ ).

Regardless of the mean differences between low and high agent status, the distributions overlapped considerably. There was also a larger difference for the female character, and the estimated status of the low-status female agent was lower compared to the male character.

For this reason, we used the perceived status instead of the dichotomous variable based on our study design.

\subsubsection{Touch perception}

The H1c hypothesis, regarding the role of social status in touch perception, was rejected-we did not find any differences between agents in Tukey's test and the only significant result was a weak correlation between pleasantness and status for the female agents (Pearson's $r=.31, p=.019$ ).

Summarising the analysis of touch perception from all three studies, we conclude that there is some evidence that gender and masculinity traits might play a role in the way agent's touch is perceived, but further studies are needed since some of the effects are small and not all predictions were confirmed.

\subsubsection{Compliance}

The pooled logistic regression model was built on 1965 observations and seven degrees of freedom, the pseudo $R^{2}=$ .28. The prediction accuracy was .79, while the accuracy for a null model was .67 (Table 2).

As in study 1 and 2, in study 3 the strongest positive effect was observed for the value of the offer. A difference in one offered coin was related to a maximal increase of chances for the acceptance of $40 \%$ (coef. $=1.60, \mathrm{SE}=.08, p<.001$ ). Moreover, for agents with a perceived social status below

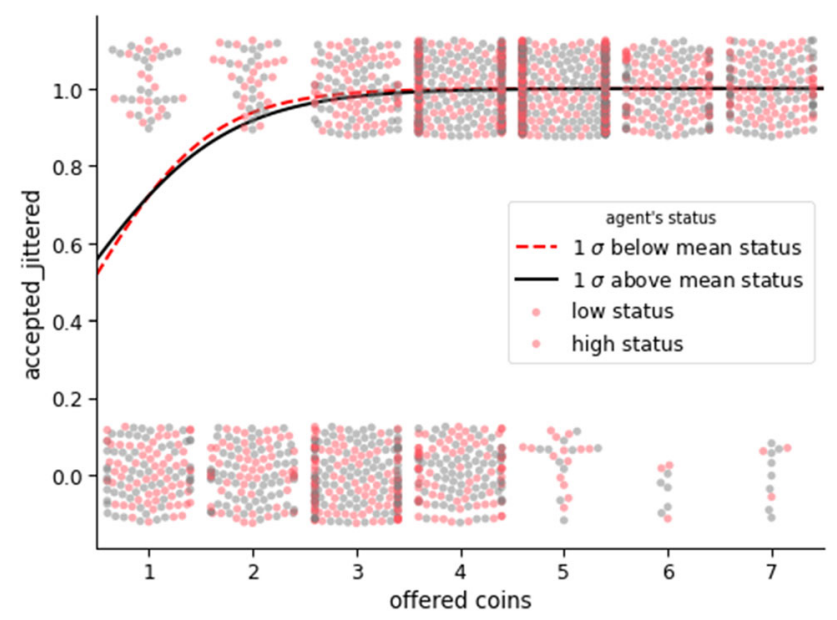

Fig. 7 The dependence on offer's value on compliance and the interaction between the offer and perceived social status (dots are coloured according to agent's designed status although we do not use it to build the model)

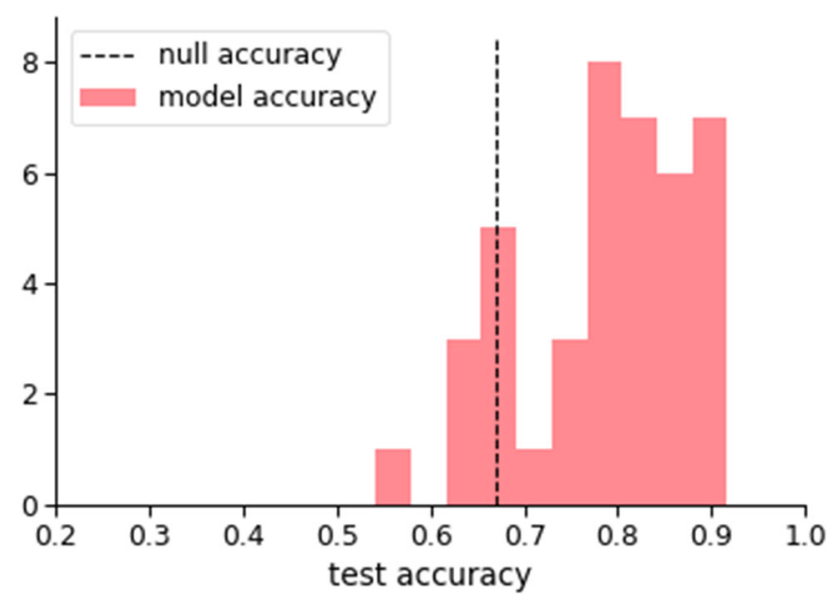

Fig. 8 Model accuracy in study 3 
average, the offer effect was weaker (coef. $=-.15, \mathrm{SE}=$ $.08, p=.051$ ) (Fig. 7).

The main positive effect of social status was also significant, with a maximal increase in chances for acceptance of $4 \%$ for a $1 \%$ increase in perceived social status (coef. $=.16$, $\mathrm{SE}=.07, p=.011$ ). We did not find any significant effects of touch nor sound. Therefore, $\mathrm{H} 4$ cannot be supported. Nevertheless, the perceived social status did influence compliance. Cross-validation showed that the patterns found on the whole dataset are stable across folds (Fig. 8, for coefficient values across CV folds, see Appendix Figure A4).

\section{Discussion}

The study aimed to analyse the influence of touch of virtual agents that varied in gender, traits stereotypically associated with gender, and social status on economic decisions. We found that even though the manipulation of the agent's characteristics was somewhat successful in all three studies, touch did not significantly increase compliance. The analysis of touch perception demonstrated some relationships between how pleasant, and how adequate touch of various agents felt. For example, in study 1, the female agent's touch felt more pleasant than the male's and its adequacy was positively related to the masculinity (though this not the case for the male agent). Moreover, in study 2, we found that the effeminate agent's touch was significantly less pleasant than the rest of the agents. Nonetheless, there was no correlation between the pleasantness or awkwardness of touch and the perceived masculinity of the agents.

Regarding the social status, the only significant relationship was a correlation between the pleasantness and perceived status for the female agents. Interestingly, all touch animations and haptic feedback for all agents were carried out in the very same manner. The agents were also not very interactive - they did not speak, maintained eye contact at random $30 / 70$ ratio, and had no facial expressions, and yet we found some significant relationships and differences. We hypothesise that the touch of more advanced embodied agents could evoke even more significant effects in their touch perception.

In all studies, the critical factor was the value of the offer made by the agent- the more generous the offer, the higher the chances of acceptance with the maximal change in chances reaching even $50 \%$ for one unit increase. These results stand in line with those of Spapé et al. [27]. In this study, the value of the offer was a strong predictor of compliance, while touch effect was much smaller-significant in comparison to no signal but insignificant in comparison to the effect of a buzz. Perhaps the Ultimatum game paradigm creates a situation of a relatively easy choice based on profit and loss account, which can be only slightly modified by any simple and unthreatening stimulus.
There was no touch effect; however, in study 1 , women accepted more offers than men and men were more affected by the perceived masculinity, which confirms gender differences in compliance. Study 2 also yielded an interactional effect between the agent's gender and the offer, suggesting that when playing with a female agent, the offer was less critical. Unfortunately, the perceived masculinity effect was not significant. H4 found the main positive effect of perceived social status. The perceived level of the agent's social status increased the chances of the offer acceptance. Perhaps the perceived social status discrepancy is more crucial than merely representing a high social status group member. It seems plausible that participants compared their own and agent's social status, and thus the more significant the discrepancy was, the more compliant they were. Moreover, Świdrak et al. [28] found a negative effect of social status importance for Polish men but only for touch trials: men for whom the social status was important accepted less likely offers after being touched. These two results together suggest that an "objective" social status matters less than our attitudes. We believe the relationship between social status and touch requires further studies which could explain the mechanism in depth.

Since the participants could have perceived the decision as a "simple" profit and loss account and players could have been primarily motivated to maximise their score, a subtle touch did not affect their decisions. The fact that they repeatedly played with each agent might have reduced the effect of touch through habituation effect, although Spapé et al. [27] did find significant effects of touch even though the game and the touch was repeated multiple times. At the same time, in other studies where repeated mediated touch was studied, it was not always significant [32, 33].

Another explanation is that participants aimed to please the experimenter [34]. To avoid the experimenter demand effect, the experimenter left the room when the game started and assured the participant about the anonymity of the collected data. From the participants' declarations collected after the debriefing, we concluded that they did not discover the aim. Nevertheless, we cannot exclude this possibility because we did not control for these factors.

Our results are in line with the literature suggesting that Polish men are less compliant with other men's requests when compared to women (study 1) [18]. Moreover, to some extent, they are influenced by perceived social status, which contributes to the literature proving that people of high social status are more influential than those of low status [15]. Our study provides partial evidence that this can also be true for embodied agents, perhaps in a situation where the decision is more complex and therefore less likely to be automatic.

These results can be discussed not only in the context of the power of touch as a social influence technique used by agents but also in the context of future agent design. With 
more and more humane agents and an increasing number of applications resulting in more diverse groups of users, developers and designers need to know what characteristics and behaviours of agents are essential for smooth humanagent communication. Here, we demonstrated that the same animation and vibration-based mediated touch could evoke different sensations or associations in participants.

The study had several shortcomings. The touch mediated by a vibrating band lacks realism. Nevertheless, social touch is quite robust and sometimes physical parameters of touch do not need to be simulated truthfully, if at all [35]. Another significant limitation is the lack of the participant's avatar. Embodiment is an essential element of building experience in virtual reality, especially one including social touch [36].

Nevertheless, the game was designed in a way to minimise gazing at one's own body. For example, the touching hand extended beyond the participant's field of view. To make sure they felt the touch realistically and that the lack of an avatar was not an issue, the experimenter asked the participants during the debriefing about their impressions. Participants reported that it was not relevant to them, and usually, that touch felt realistic and natural which confirms the vibration gave a sufficiently realistic impression and that participants were simply too focused on increasing their score in the game.

Due to budget limitations, we were not able to remove these and other minor problems with the procedure, nor collect more data, which might have affected the power of the experiments.

Future studies should explore further the efficiency of an agent's touch on various kinds of behaviour, including spontaneous and altruistic help. The lack of effect of touch found in our study and the small effect sizes of touch in Spapé et al. [27] suggest that economic decisions may be relatively resistant to some social influence techniques. It is crucial to understand in which circumstances mediated touch can modulate behaviour in immersive virtual reality and when it cannot. We expect that soon money transactions might be available directly in VR games and applications with embodied agents offering services and goods. How we use touch in such contexts can play an essential role in the commercial success of these products.

A second, equally important line of future research, seems to be the social context of embodied agents. In order to build a more inclusive and open immersive VR community, we need to investigate deeper how stereotypes and prejudice influence user-agent interaction and how can it be improved.

To conclude, our study demonstrates that the influence of social cues, like gender, masculinity/femininity, or social status, on the efficiency of touch seems to be subtle, implicit, transitory, and challenging to grasp. We believe that with the advancement and a growing number of applications of the technology in various fields and social groups, the ques- tion of what kind of agents (including their gender, physical appearance, nonverbal and verbal communication, and culturally sensitive traits) should be used remains more critical than ever.

Acknowledgements We want to thank Prof. Dariusz Doliński for his valuable comments on the study design and Martyna Radzikowska and Marta Siepsiak who took care of the recruitment process and collected part of the data.

Author's contribution Authors JŚ and GP contributed to the study conception and design. Material preparation and data collection were performed by JŚ. Data analysis was carried out by JŚ and AI. AI carried out the analysis of compliance based on the logistic regression model. The first draft of the manuscript was written by JŚ, and all authors commented on the manuscript. All three authors read and approved the final manuscript.

Funding This study was funded by the National Centre of Science in Poland (No. 2014/15/N/HS6/04135).

\section{Compliance with ethical standards}

Conflict of interest The authors declare that they have no conflict of interest.

\section{References}

1. Gudykunst WB (2003) Cross-cultural and intercultural communication. SAGE, Thousand Oaks

2. Baugher AR, Gazmararian JA (2015) Masculine gender role stress and violence: a literature review and future directions. Aggress Violent Behav 24:107-112. https://doi.org/10.1016/j.avb.2015.04. 002

3. de Lemus S, Spears R, Bukowski M et al (2013) Reversing implicit gender stereotype activation as a function of exposure to traditional gender roles. Soc Psychol 44:109-116. https://doi.org/10.1027/18 64-9335/a000140

4. Rudman LA, Glick P (2001) Prescriptive gender stereotypes and backlash toward Agentic women. J Soc Issues 57:743-762. https:// doi.org/10.1111/0022-4537.00239

5. Allbeck J, Badler N (2002) Toward representing agent behaviors modified by personality and emotion. In: Embodied conversational agents at AAMAS, pp 15-19

6. Koda T, Ishioh T, Watanabe T, Kubo Y (2017) Perception of masculinity and femininity of agent's appearance and self-adaptors. In: van den Herik J, Filipe J (eds) Agents and artificial intelligence. Springer International Publishing, Cham, pp 3-18

7. Henley NM (1973) Status and sex: some touching observations. Bull Psychon Soc 2:91-93. https://doi.org/10.3758/BF03327726

8. Roese NJ, Olson JM, Borenstein MN et al (1992) Same-sex touching behavior: the moderating role of homophobic attitudes. J Nonverbal Behav 16:249-259. https://doi.org/10.1007/BF014620 05

9. Floyd K (2000) Affectionate same-sex touch: the influence of homophobia on observers' perceptions. J Soc Psychol 140:774-788. https://doi.org/10.1080/00224540009600516

10. Crusco AH, Wetzel CG (1984) The Midas touch: the effects of interpersonal touch on restaurant tipping. Pers Soc Psychol Bull 10:512-517. https://doi.org/10.1177/0146167284104003 
11. Guéguen N, Afifi F, Brault S et al (2011) Failure of tactile contact to increase request compliance: the case of blood donation behavior. J Articles Support Null Hypothesis JASNH 8:1-8

12. Hornik J (2010) Tactile stimulation and consumer response. J Consum Res 19:449-458. https://doi.org/10.1086/209314

13. Pausell S, Goldman M (1984) The effect of touching different body areas on prosocial behavior. J Soc Psychol 122:269-273

14. Brockner J, Pressman B, Cabitt J, Moran P (1982) Nonverbal intimacy, sex, and compliance: a field study. J Nonverbal Behav 6:253-258. https://doi.org/10.1007/BF00987192

15. Guéguen N (2002) Status, apparel and touch : their joint effects on compliance to a request. North Am J Psychol 4:279-286

16. Brown N (2019) Nick Brown's blog: an update on our examination of the research of Dr. Nicolas Guéguen. In: Nick Brown's blog. https://steamtraen.blogspot.com/2019/05/an-update-on-ourexamination-of.html. Accessed 19 May 2020

17. Europe ILGA (2020) Annual review of the human rights situation of Lesbian, Gay, Bisexual, Trans and Intersex People in Europe and Central Asia. ILGA-Europe, Brussels

18. Doliński D (2010) Touch, compliance, and homophobia. J Nonverbal Behav 34:179-192. https://doi.org/10.1007/s10919-010-00901

19. Doliński D (2013) Male homophobia, touch, and compliance: a matter of the touched, not the toucher. Polish Psychol Bull 44:457-461. https://doi.org/10.2478/ppb-2013-0051

20. Camps J, Tuteleers C, Stouten J, Nelissen J (2013) A situational touch: how touch affects people's decision behavior. Soc Influence 8:237-250. https://doi.org/10.1080/15534510.2012.719479

21. MCcluskey M (2020) The coronavirus outbreak keeps humans from touching. Here's why that's so stressful. Time

22. Huisman G, Kolkmeier J, Heylen D (2014) With us or against us: simulated social touch by virtual agents in a cooperative or competitive setting. In: Bickmore T, Marsella S, Sidner C (eds) Intelligent virtual agents. Springer International Publishing, Cham, pp 204-213

23. Hoppe M, Rossmy B, Neumann DP, et al (2020) A human touch: social touch increases the perceived human-likeness of agents in virtual reality. In: Proceedings of the 2020 CHI conference on human factors in computing systems. Association for Computing Machinery, Honolulu, HI, USA, pp 1-11

24. Güth W, Schmittberger R, Schwarze B (1982) An experimental analysis of ultimatum bargaining. J Econ Behav Organ 3:367-388. https://doi.org/10.1016/0167-2681(82)90011-7

25. Güth W, Kocher MG (2014) More than thirty years of ultimatum bargaining experiments: motives, variations, and a survey of the recent literature. J Econ Behav Organ 108:396-409. https://doi. org/10.1016/j.jebo.2014.06.006
26. Nouri E, Traaum D (2014) Cultural differences in playing repeated ultimatum game online with virtual humans. In: 2014 47th Hawaii international conference on system sciences. IEEE, Waikoloa, HI, pp 1213-1220

27. Spapé MM, Hoggan EE, Jacucci G, Ravaja N (2015) The meaning of the virtual Midas touch: an ERP study in economic decision making. Psychophysiology 52:378-387. https://doi.org/10.1111/psyp. 12361

28. Świdrak J, Pochwatko G, Navarro X et al (2020) The joint influence of social status and personal attitudes in a contact and open versus a noncontact and homophobic culture on the virtual Midas touch. Virtual Real 24:619-633. https://doi.org/10.1007/s10055-019-004 23-8

29. Gallace A, Spence C (2010) The science of interpersonal touch: an overview. Neurosci Biobehav Rev 34:246-259. https://doi.org/10. 1016/j.neubiorev.2008.10.004

30. Turnbull PRK, Phillips JR (2017) Ocular effects of virtual reality headset wear in young adults. Sci Rep 7:1-9. https://doi.org/10.10 38/s41598-017-16320-6

31. Gelman A, Hill J (2006) Logistic regression. Data analysis using regression and multilevel/hierarchical models. Cambridge University Press, Cambridge, pp 79-108

32. Harjunen VJ, Spapé M, Ahmed I et al (2018) Persuaded by the machine: the effect of virtual nonverbal cues and individual differences on compliance in economic bargaining. Comput Hum Behav 87:384-394. https://doi.org/10.1016/j.chb.2018.06.012

33. Spapé M, Harjunen V, Ahmed I et al (2019) The semiotics of the message and the messenger: how nonverbal communication affects fairness perception. Cogn Affect Behav Neurosci 19:1259-1272. https://doi.org/10.3758/s13415-019-00738-8

34. Zizzo DJ (2008) Experimenter demand effects in economic experiments. Social Science Research Network, Rochester

35. van Erp JBF, Toet A (2015) Social touch in human-computer interaction. Front Digit Humanit 2:2. https://doi.org/10.3389/fdigh.201 5.00002

36. Kilteni K, Groten R, Slater M (2012) The sense of embodiment in virtual reality. Presence Teleoper Virtual Environ 21:373-387. https://doi.org/10.1162/PRES_a_00124

Publisher's Note Springer Nature remains neutral with regard to jurisdictional claims in published maps and institutional affiliations. 